DOI: 10.12957/demetra.2016.19217

\title{
Percepção da importância e adesão ao tratamento dietético de pacientes com doença inflamatória intestinal
}

\author{
Perception of the importance and accession to the patient with dietary treatment \\ inflammatory bowel disease
}

\author{
Cristina Henschel de Matos' \\ Anny Caroline Lopes de Paulo? \\ Samara Ferreira de Souza de Carvalho' \\ Izabella Imianovsky' \\ Vanessa Imianowsky' \\ Claiza Barretta' \\ Luciane Peter Grillo?
}

\footnotetext{
1 Universidade do Vale do Itajaí, Centro de Ciências da Saúde, Curso de Nutrição, Curso de Nutrição. Itajaí-SC, Brasil.
}

Correspondência / Correspondence

Cristina Henschel de Matos

E-mail:matoscris@gmail.com

\section{Resumo}

As doenças inflamatórias intestinais (DII), representadas pela doença de Crohn e retocolite ulcerativa, resultam em modificações no consumo alimentar e alterações nutricionais. O objetivo deste trabalho foi avaliar a percepção da importância e a adesão ao tratamento dietético de pacientes com DII. A população foi composta por pacientes do ambulatório interdisciplinar de DII da Unidade de Saúde Familiar e Comunitária de Itajaí, SC. Aplicou-se um questionário semiestruturado contendo dados de identificação, qualidade do atendimento e adesão às orientações nutricionais contidas no protocolo de atendimento das nutricionistas. Foi avaliado o estado nutricional através das medidas antropométricas e do diagnóstico nutricional no início do tratamento e no momento da coleta de dados.Para análise estatística, utilizou-se o programa STATA 13.0. Dos 56 entrevistados, $98 \%$ consideram importante receber orientações sobre alimentação e $93 \%$ afirmaram que as orientações nutricionais auxiliaram no tratamento da doença. Houve prevalência de excesso de peso em $52 \%$ dos avaliados após o acompanhamento com a nutrição, com diminuição da desnutrição. Mais de $70 \%$ dos pacientes aderiram às orientações, sendo que a média de orientações aderidas para melhora do IMC foi de 7,61, e para a percepção de auxílio no tratamento foi de 7,69. A orientação de maior adesão foi "diminuir o consumo de gorduras" e a de menor, "consumir somente o caldo do feijão". Diante dos resultados, observou-se o reconhecimento da importância do atendimento nutricional e sua necessidade para o controle dos sintomas, bem como a melhora do estado nutricional e adesão de grande parte da conduta dietética.

Palavras-chave: Colite Ulcerativa. Doença de Crohn. Estado Nutricional. Dietoterapia. 


\section{Abstract}

Inflammatory bowel diseases (IBD), represented by Crohn's disease and ulcerative colitis, result in changes in food consumption and nutritional changes. This paper aimed to assess the perception of importance and compliance with diet therapy of patients with IBD. Sample was made up by patients of the IBD multidisciplinary clinic of a family and community health unit in the town of Itajaí, Santa Catarina state, Brazil. A semi-structured questionnaire was applied to patients with IBD, containing questions on personal data, quality of care provided, and their compliance with the nutritional guidelines provided in the care protocol given by the nutritionists. The patient's nutritional status was also evaluated, through anthropometric measurements and nutritional diagnosis at beginning of treatment and at the time of data collection. Statistical analyses were performed using the software STATA 13.0. Of the 56 patients interviewed, 98\% considered it important to receive guidelines on food, and $93 \%$ said that their adhesion to the guidelines helped treating the disease. There was a prevalence of overweight patients, with $52 \%$ after follow-up with nutritional guidance, and reduction of malnutrition. Over $70 \%$ of patients said they had complied with the guidelines, and the average adherence to guidelines for BMI was 7.61 and for perception of aid it was 7.69. The guideline with highest adherence was "reducing consumption of fats" and the least adhered to was "consume only bean broth". Based on the results, we observed that patients recognize the importance of nutritional care and its importance in controlling symptoms, as well as the improvement in nutritional status and adhesion to most of the diet therapy guildelines.

Key words: Ulcerative Colitis. Crohn's Disease. Nutritional Status. Diet Therapy.

\section{Introdução}

A doença inflamatória intestinal (DII), representada pela doença de Crohn (DC) e pela retocolite ulcerativa idiopática (RCUI), é um grupo de condições inflamatórias crônicas, resultado de ativação persistente inadequada do sistema imune mucoso. ${ }^{1}$ A DC define-se como um processo inflamatório crônico, idiopático e transmural que afeta um ou mais segmentos do tubo digestivo. A RCUI também é um processo inflamatório crônico idiopático que atinge o reto, estendendo-se contínua e proximalmente, podendo atingir a totalidade do cólon. ${ }^{2}$ 
A etiologia das DIIs está relacionada a aspectos genéticos, imunológicos e ambientais. Dentre os aspectos ambientais, encontram-se os fatores dietéticos. Sugere-se que dietas com baixo teor de fibras e alto conteúdo de açúcar, gordura animal, gorduras totais, ácidos graxos poliinsaturados ômega-6 e carnes podem constituir fatores de risco para estas doenças. Propõe-se também que o consumo de sucos, frutas cítricas e vegetais pode reduzir o risco de desenvolvimento tanto de DC quanto de RCUI. ${ }^{3}$

Diversos estudos epidemiológicos vêm demonstrando incidência crescente da DC e da RCUI nas últimas décadas. As DIIs acometem pessoas de diferentes classes socioeconômicas, idade, sexo e nacionalidade. ${ }^{4}$ Ocorrem em todo o mundo e representam sério problema de saúde, uma vez que atingem em sua maioria pessoas jovens, cursam com recidivas frequentes e assumem formas clínicas de alta gravidade. ${ }^{5}$

O Brasil ainda é considerado área de baixa prevalência de DIIs, apesar do aumento significativo da incidência destas doenças nos registros da literatura nacional. Em nosso meio, estas doenças não são consideradas de notificação compulsória, o que leva a pensar que as DIIs podem não estar sendo diagnosticadas, uma vez que os prontuários médicos registram altas taxas de diarreias de etiologia bacteriana ou parasitária. ${ }^{5}$

De acordo com Salviano, Burgos \& Santos, ${ }^{6}$ as DIIs são comumente associadas à desnutrição proteico-energética, como também à carência de micronutrientes tanto em pacientes hospitalizados como em ambulatório, causadas por redução da ingestão alimentar, má absorção, aumento das perdas gastrointestinais e necessidades nutricionais aumentadas. Tais alterações têm impacto em todas as idades, sofrendo a influência da fase de atividade, extensão e localização da doença. A desnutrição, no entanto, agrava o prognóstico tanto do paciente em tratamento clínico, quanto naqueles submetidos a cirurgias, além de deteriorar a competência imune.

O tratamento nutricional na vigência das DIIs tem como objetivo o controle dos sintomas, a prevenção e a correção da desnutrição e das diversas deficiências nutricionais e a redução das sequelas em longo prazo. ${ }^{3}$ Além disso, suporte nutricional adequado deve ser instituído para poder reduzir as indicações cirúrgicas e as complicações operatórias, mantendo e/ou recuperando as condições nutricionais. ${ }^{6}$

Sendo assim, é de suma importância conhecer as condições dos pacientes acometidos pelas DIIs e as dificuldades na adesão ao tratamento nutricional, podendo fornecer mais subsídios para o trabalho da equipe multiprofissional que atua no Ambulatório de Doença Inflamatória Intestinal da Unidade de Saúde Escola de Itajaí.

O objetivo do presente estudo foi avaliar a percepção da importância e a adesão ao tratamento dietético de pacientes portadores de doença inflamatória intestinal. 


\section{Método}

Trata-se de estudo de caráter transversal e descritivo, realizado no ambulatório multiprofissional de DIIs de uma Unidade de Saúde Escola de Itajaí-SC, de março de 2014 a junho de 2015. O estudo foi aprovado pelo Comitê de Ética em Pesquisa da UNIVALI, sob o parecer número 554.518, de 12 de março de 2014.

A coleta de dados foi realizada a partir de um questionário semiestruturado, composto de questões abertas e fechadas, aplicado em forma de entrevista durante a espera ou após o término das consultas em local privativo, calmo e tranquilo. Os critérios de inclusão adotados foram: diagnóstico confirmado das doenças, realização de pelo menos uma consulta com a nutrição, pacientes menores de 18 anos somente acompanhados dos pais ou responsáveis e aceite em participar da pesquisa, mediante assinatura do Termo de Consentimento Livre e Esclarecido (TCLE).

Quanto aos fatores de exclusão, não puderam participar da pesquisa indivíduos nas seguintes condições: ter realizado consulta ou recebido orientação nutricional sobre DII por nutricionista que não pertença ao ambulatório multiprofissional, apresentar condição psiquiátrica ou neurocognitiva que impeça a obtenção de dados clínicos fidedignos (definida pelo julgamento clínico dos investigados); gravidez ou lactação; apresentar dificuldade de alimentação via oral, ser cadeirante; ter realizado gastroplastia ou transplante de órgão e apresentar diagnóstico de insuficiência renal com indicação de diálise.

O questionário de avaliação continha 35 questões divididas em dados de identificação, estado nutricional antes e após consulta com nutricionista, dados sobre a doença, questões relacionadas ao atendimento ambulatorial e sobre a adesão às principais orientações nutricionais fornecidas pelas nutricionistas da equipe aos pacientes com DII. Informações sobre diagnóstico da doença, tempo de atendimento ambulatorial, peso e estatura foram obtidas nos prontuários dos pacientes. O diagnóstico nutricional foi feito utilizando o indicador índice de massa corporal (IMC) e a classificação de acordo com os critérios propostos pelo Ministério da Saúde. ${ }^{7}$ Para fins estatísticos, considerou-se orientação aderida aquelas que apresentaram um percentual de adesão por todos os pacientes avaliados de $70 \%$ ou mais.

Quanto a análise e interpretação dos resultados, os dados coletados na pesquisa foram tabulados com o auxílio dos programas Microsoft Excel $^{\circledR} \mathrm{e} \mathrm{Word}^{\circledR}{ }^{\circledR}$ a análise dos dados foi realizada confrontando os resultados com a literatura recente sobre o assunto e a análise estatística foi feita com auxílio do programa STATA 13.0. Foram calculados para as variáveis com distribuição simétrica a média e o desvio padrão (apresentado no formato média +/- desvio padrão). Para as variáveis categóricas, foram calculadas as frequências absolutas e relativas (número e \%). A associação entre as variáveis categóricas foi testada por meio do teste do qui-quadrado de Pearson e do teste exato de Fisher, e a diferença entre as médias foi calculada através do teste $t$ Student para amostras dependentes. 
Foram considerados como possíveis confundidores a idade de diagnóstico, tempo de diagnóstico, escolaridade, tempo de tratamento com a nutrição e número de orientações aderidas. As análises ajustadas foram conduzidas a partir da regressão linear múltipla. Em todas as análises, assumiuse um nível de significância de 5\%.

\section{Resultados e Discussão}

No período do estudo, estavam cadastrados no ambulatório de DIIs 88 pacientes, dos quais 56 foram atendidos entre os meses de março de 2014 a junho de 2015, e correspondiam aos critérios de inclusão.

Os resultados demonstraram que $57 \%$ ( $\mathrm{n}=32$ ) de indivíduos atendidos eram do gênero feminino e 43\% ( $\mathrm{n}=24)$ do gênero masculino. A média de idade foi de 46 $\pm 15,29$ anos, variando entre $14 \mathrm{e}$ 72 anos. Em um estudo similar, Silva, Schieferdecker \& Amarante $^{8}$ avaliaram 55 pacientes com DII, sendo que $58 \%(\mathrm{n}=32)$ eram do sexo feminino e na faixa etária dos 40 anos. No entanto, a literatura mostra que as DIIs não possuem distinção de sexo, ${ }^{9}$ mas sabe-se que a tendência é as mulheres procurarem mais os serviços de saúde do que os homens, ${ }^{10}$ seja por prevenção ou para recuperação, podendo assim justificar a prevalência de mulheres nesta pesquisa.

Quanto à idade dos pacientes, a DII costuma manifestar-se frequentemente em adolescentes e jovens adultos, mas pode ocorrer em qualquer idade, ${ }^{11}$ fato confirmado pelo presente estudo, uma vez que para os pacientes avaliados a média de idade em que houve o diagnóstico de DII foi $39 \pm 15,9$ anos. Entretanto, segundo relato dos próprios pacientes, algumas manifestações ocorreram anos antes do diagnóstico.

Quanto à escolaridade, identificou-se média de oito $( \pm 4,2)$ anos de estudo e apenas $55 \%(\mathrm{n}=31)$ trabalhavam rotineiramente; os demais estavam desempregados, eramaposentados ou afastados. Verificou-se, ainda, que $68 \%(n=38)$ declararam-se casados e $95 \%(n=53)$ conviviam com a família, composta por em média $3( \pm 1,47)$ pessoas. Estes achados podem ser decisivos para a recuperação do paciente, já que, segundo Salviano, Burgos \& Santos, ${ }^{6}$ a proximidade da família está relacionada ao retorno mais rápido ao estilo de vida normal, após períodos agudos da doença, tendo resultados positivos na recuperação dos pacientes.

Em relação à renda familiar, a média relatada pelo total de pacientes entrevistados foi de aproximadamente $\mathbf{R} \$ 2.700,00( \pm 1.753,82)$, variando de 700 a 10.000 reais, e renda per capita de R \$ 900,00. Em contrapartida, no estudo de Salviano, Burgos \& Santos, ${ }^{6}$ a maioria dos pacientes entrevistados apresenta renda familiar inferior a dois salários mínimos, o que seria incompatível com a manutenção de um estado nutricional adequado. 
O estado nutricional nas DIIs pode ser afetado por redução na ingestão alimentar causada pelos sintomas gastrintestinais, má absorção pela diminuição da área absortiva, deficiência de sais biliares e pelo tratamento medicamentoso, com maiores alterações na fase aguda da doença. ${ }^{8}$ Estudos recentes mostraram que muitos pacientes em remissão estão em bom estado nutricional e outros com sobrepeso, sendo necessário o acompanhamento com a nutricionista. ${ }^{12}$

A figura 1 apresenta a evolução dos resultados referentes à avaliação do estado nutricional antes e após o acompanhamento nutricional no ambulatório de DIIs, que em média foi de 893 $( \pm 506,57)$ dias.
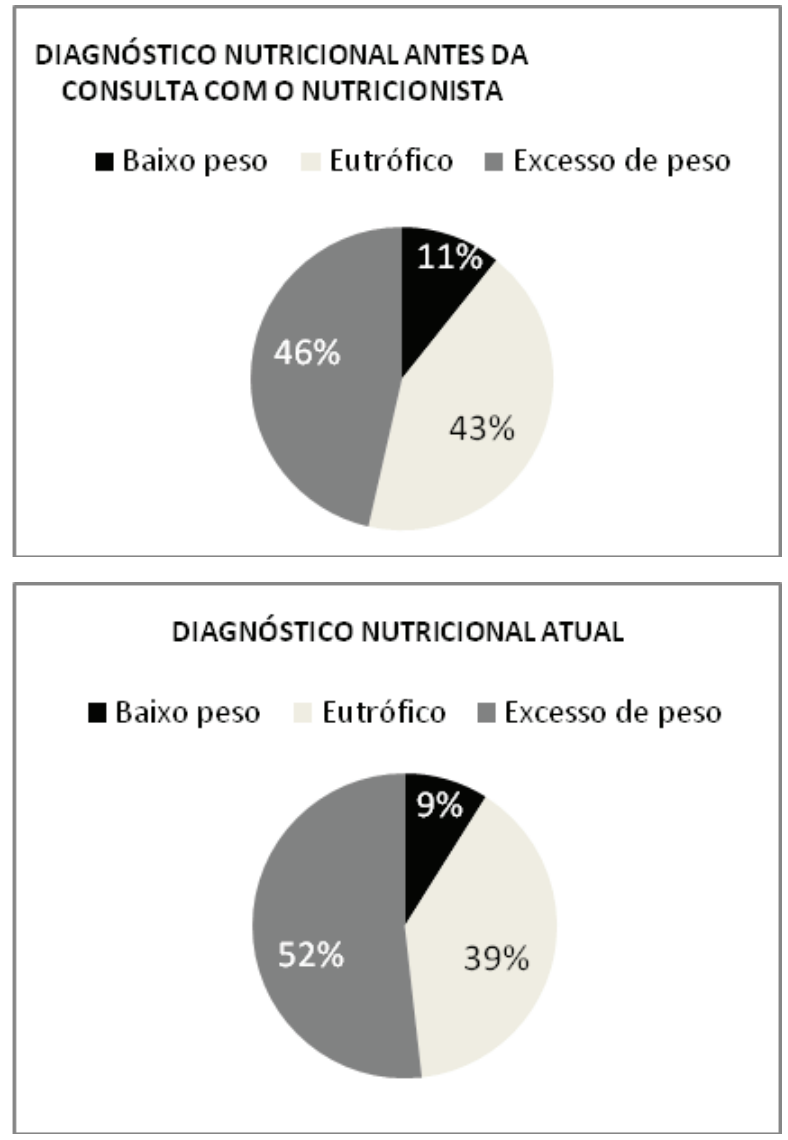

Figura 1. Comparação do estado nutricional dos pacientes antes e após atendimento nutricional no ambulatório multidisciplinar de Doença Inflamatória Intestinal. Itajaí-SC, junho, 2014. 
De acordo com a figura 1, é possível observar que, no decorrer do tratamento, houve redução de $18,8 \%$ da desnutrição e aumento de $13,04 \%$ de pacientes com sobrepeso. Deste modo, além de melhorar a qualidade de vida, acredita-se que as orientações nutricionais, quando aderidas, podem melhorar a condição antropométrica dos pacientes, mas quando não aderidas na remissão da doença, podem ocasionar o sobrepeso.

Não foram observadas diferenças de significativas entre o peso $(p=0,977)$ e o IMC $(p=0,971)$ antes e após consulta nutricional. Os resultados das análises mostraram que os valores, quando ajustados para as variáveis "idade de diagnóstico", "tempo de diagnóstico", "escolaridade", "tempo de tratamento nutricional" e "número de orientações aderidas" apresentaram somente associação entre IMC final e idade de diagnóstico $(\beta=0,13 ; \mathrm{IC}=0,04$ a 0,22$)(\mathrm{p}=0,005)$.

Estudo realizado por Silva, Schieferdecker \& Amarante ${ }^{9}$ demonstrou que os pacientes já em remissão apresentaram maior ingestão de carboidratos e menor de proteína, quando comparados com controles saudáveis. Isso provavelmente se deve à redução dos sintomas, o que fez com que os indivíduos acabassem se alimentando em maior quantidade e até mesmo perdendo o medo de se alimentar. No entanto, Boutros \& Maron $^{13}$ afirmam que, nas duas últimas décadas, a obesidade está se tornando cada vez mais frequente entre os pacientes com DIIs.

Em relação ao diagnóstico da doença, $54 \%(n=30)$ dos pacientes apresentavam doença de Crohn e $46 \%$ (n=26) retocolite ulcerativa, diagnosticada em média há seis anos $( \pm 6,57)$, variando de três meses a 30 anos. Pode-se observar maior número de pacientes com DC, havendo porém pouca diferença quanto à presença de RC, como no estudo de Israeli et al., ${ }^{14}$ no qual foram entrevistados 244 pacientes com DII, sendo 52\% DC e 48\% RC.

Observou-se também que $44 \%$ (n=25) dos indivíduos apresentavam intolerância a lactose (IL) e $60 \%$ (n=34) a hábitos intestinais irregulares. A IL pode ser justificada pelo comprometimento da mucosa intestinal, mais comum na DC, que pode levar à redução da atividade da enzima lactase, devido à diminuição do tamanho do enterócito, do núcleo e da borda em escova, ocasionado pela diarreia. $^{15}$

Quanto aos sintomas relatados, predominou a flatulência $(85 \%$; $n=48)$, seguido de dor abdominal (55\%; $\mathrm{n}=31$ ) e distensão abdominal $(46 \% ; \mathrm{n}=26)$, havendo relatos de hematoquezia $(39 \% ; n=22)$ e febre $(19 \% ; n=11)$. Com base na literatura, observou-se que os sintomas causados em intolerantes à lactose e em paciente com DII são similares, dentre os quais diarreia, flatulência e dor abdominal. ${ }^{15}$

Apesar de não haver alteração nas necessidades energéticas, observa-se que existe inadequação na ingestão alimentar em pacientes com DII, tanto em atividade como em remissão da doença. ${ }^{8}$ 
Quando a doença se encontra em atividade, é importante que a alimentação auxilie no controle dos sintomas e previna ou reverta à perda de peso através do uso de suplementos nutricionais adequados. Deve haver restrição de carboidratos simples e alimentos que causam flatulência, restringindo também o teor de fibras insolúveis e resíduos, fracionando a alimentação, contendo poucos volumes. ${ }^{3}$ Com a melhora e o início da fase de remissão, a dieta deve ser balanceada e as calorias devem ser adequadas ao estado nutricional do paciente, acrescentando aos poucos as fibras e mantendo o teor de gordura moderado. ${ }^{8}$ Diante do exposto, é fundamental que o acompanhamento nutricional seja realizado em todas as fases da doença.

O quadro 1 apresenta os resultados relacionados à opinião dos pacientes quanto ao atendimento nutricional prestado pelas nutricionistas da equipe multiprofissional do Ambulatório de DIIs.

Quadro 1. Opinião dos pacientes sobre o atendimento nutricional oferecido no ambulatório multidisciplinar de Doença Inflamatória Intestinal. Itajaí-SC, junho, 2014.

\begin{tabular}{|l|c|c|c|c|c|c|c|c|}
\hline \multirow{2}{*}{} & \multicolumn{2}{|c|}{ Sim } & \multicolumn{2}{c|}{ Não } & \multicolumn{2}{c|}{ Parcial } & \multicolumn{2}{c|}{ Não sei } \\
\cline { 2 - 9 } & $\mathrm{N}$ & $\%$ & $\mathrm{~N}$ & $\%$ & $\mathrm{~N}$ & $\%$ & $\mathrm{~N}$ & $\%$ \\
\hline $\begin{array}{l}\text { Recebeu orientação sobre } \\
\text { alimentação desde o início }\end{array}$ & 52 & 93 & 3 & 5 & 1 & 2 & - & - \\
\hline $\begin{array}{l}\text { Compreendeu as } \\
\text { orientações }\end{array}$ & 52 & 93 & - & - & 4 & 7 & - & - \\
\hline $\begin{array}{l}\text { Acha importante } \\
\text { recebê-las junto com o } \\
\text { atendimento médico }\end{array}$ & 55 & 98 & - & - & 1 & 2 & - & - \\
\hline $\begin{array}{l}\text { Acha que as orientações } \\
\text { nutricionais auxiliaram no } \\
\text { tratamento da doença }\end{array}$ & 52 & 93 & 1 & 2 & 1 & 2 & 2 & 3 \\
\hline
\end{tabular}

De acordo com o quadro 1, 98\% (n=55) dos pacientes entrevistados consideram importante receber orientações sobre alimentação em conjunto com o atendimento médico. Além disso, a maioria $(93 \%, \mathrm{n}=52)$ as compreendeu e afirma que sua adesão auxiliou no tratamento da doença. Sabe-se que a adesão ao tratamento, seja ele nutricional ou médico, desempenha papel importante no sucesso do mesmo. É um processo multifatorial que se estabelece mediante a parceria entre o profissional da saúde e o paciente, abrangendo aspectos que compreendem frequência dos atendimentos, reconhecimento, aceitação e adaptação da sua condição de saúde, identificação de hábitos de risco, preservação de atitudes que promovam a qualidade de vida, desenvolvimento da 
consciência para o autocuidado e manutenção da busca de saúde. Doentes crônicos têm menor adesão ao tratamento, visto que os esquemas terapêuticos, muitas vezes complexos, exigem imenso esforço do paciente e devem ser seguidos constantemente. ${ }^{16}$

Os dados referentes à adesão das principais orientações nutricionais voltadas à DII são apresentados no quadro 2.

Quadro 2. Avaliação da adesão das orientações nutricionais fornecidas a pacientes do ambulatório multidisciplinar Doença Inflamatória Intestinal. Itajaí-SC, junho, 2014.

\begin{tabular}{|c|c|c|c|c|c|c|}
\hline \multirow[t]{2}{*}{$\begin{array}{c}\text { Principais orientações } \\
\text { nutricionais }\end{array}$} & \multicolumn{2}{|c|}{$\begin{array}{l}\text { Sempre/quase } \\
\text { sempre }\end{array}$} & \multicolumn{2}{|c|}{$\begin{array}{l}\text { Raramente/ } \\
\text { nunca }\end{array}$} & \multicolumn{2}{|c|}{$\begin{array}{c}\text { Não recebeu } \\
\text { essa orientação }\end{array}$} \\
\hline & $\mathrm{N}$ & $\%$ & $\mathrm{~N}$ & $\%$ & $\mathrm{~N}$ & $\%$ \\
\hline $\begin{array}{l}\text { 1. Realizar refeições em local } \\
\text { calmo/tranquilo }\end{array}$ & 44 & 78 & 10 & 18 & 2 & 4 \\
\hline 2. Realizar de 4 a 6 refeições/dia & 42 & 75 & 14 & 25 & - & - \\
\hline $\begin{array}{l}\text { 3. Evitar alimentos irritativos } \\
\text { da mucosa }\end{array}$ & 44 & 78 & 10 & 18 & 2 & 4 \\
\hline $\begin{array}{l}\text { 4. Evitar alimentos que } \\
\text { provocam gases }\end{array}$ & 36 & 64 & 14 & 25 & 6 & 11 \\
\hline $\begin{array}{l}\text { 5. Consumir frutas, legumes e } \\
\text { verduras cozidos }\end{array}$ & 44 & 78 & 10 & 18 & 2 & 4 \\
\hline $\begin{array}{l}\text { 6. Diminuir o consumo de } \\
\text { frituras/gorduras }\end{array}$ & 52 & 93 & 3 & 5 & 1 & 2 \\
\hline $\begin{array}{l}\text { 7. Evitar produtos } \\
\text { industrializados }\end{array}$ & 44 & 78 & 10 & 18 & 2 & 4 \\
\hline $\begin{array}{l}\text { 8. Diminuir o consumo de leites } \\
\text { e derivados }\end{array}$ & 25 & 44 & 29 & 52 & 2 & 4 \\
\hline $\begin{array}{l}\text { 9. Consumir somente o caldo } \\
\text { do feijão }\end{array}$ & 21 & 37 & 23 & 41 & 12 & 22 \\
\hline $\begin{array}{l}\text { 10. Evitar consumo excessivo de } \\
\text { café e chimarrão }\end{array}$ & 29 & 52 & 21 & 37 & 6 & 11 \\
\hline $\begin{array}{l}\text { 11. Reduzir o consumo de } \\
\text { açúcares e doces }\end{array}$ & 41 & 73 & 13 & 23 & 2 & 4 \\
\hline
\end{tabular}


Observou-se que das 11 recomendações avaliadas, $64 \%(n=7)$ foram aderidas por mais de $70 \%$ dos pacientes, embora não se tenha encontrado associação entre a adesão das orientações e diagnóstico nutricional $(\mathrm{p}=0,359)$. Verificou-se que a média de orientações necessárias para que os pacientes melhorassem o IMC (aproximação do IMC ideal para a faixa etária) e tivessem a percepção que a orientação nutricional auxiliou no tratamento foi de 7,61 e 7,69, respectivamente. Destaca-se que a orientação mais realizada foi "diminuir o consumo de gorduras e frituras". Já aquelas que mais apresentaram dificuldades de adesão foram: "consumir somente o caldo do feijão", "diminuir o consumo de leites e derivados" e "evitar o consumo excessivo de café e chimarrão".

Em relação às orientações mais aderidas, a diminuição da ingestão de gorduras e frituras (93\%) e de alimentos industrializados (78\%) foram achados importantes sobre as orientações alimentares, principalmente quando comparados aos resultados de Campos \& Cavalcante, ${ }^{11}$ que demonstraram, em seu estudo, que $83 \%$ dos pacientes com DC costumam consumir frituras e $48 \%$ alimentos enlatados. Sabe-se que, quando há inflamação da mucosa do intestino, a absorção de gorduras é dificultada pela redução da área absortiva, podendo agravar a estearorreia. ${ }^{17}$

Corroborando esses resultados, está a adesão $(78 \%$; n=44) à recomendação para evitar alimentos irritativos da mucosa, já que o consumo destes alimentos estimula o aumento do ácido gástrico, ocasionando irritações constantes. ${ }^{18}$ Segundo Santos, Silva \& Santana, ${ }^{19}$ a redução destes alimentos pode também estar relacionada ao medo do aparecimento ou da piora dos sintomas.

A orientação para realizar refeições em local calmo e tranquilo teve taxa de adesão alta (78\%, $\mathrm{n}=44)$ e o fracionamento das refeições, aderido por $75 \%(\mathrm{n}=42)$ dos pacientes, pode auxiliar no controle da concentração sérica de glicose, do colesterol, na manutenção do peso corporal adequado e também na saúde intestinal. ${ }^{20}$

A adesão de $78 \%$ (n=44) dos pacientes ao consumo de frutas, legumes e verduras cozidas melhora a qualidade da alimentação e ajuda a minimizar sintomas, sobretudo na fase ativa da doença. Segundo Santos, ${ }^{21}$ o consumo adequado de vegetais e frutas contribui para a diminuição do risco de desenvolvimento de DII. Somado a isto, a redução do consumo de açúcares e doces, aderido por $73 \%(\mathrm{n}=41)$ dos pacientes é um achado significativo, pois para Almeida et al. ${ }^{22}$ o consumo destes alimentos pode causar flatulência e diarreia, prejudicando amicrobiota gastrointestinal.

Em contrapartida, o baixo percentual de adesão das orientações "evitar alimentos que provocam gases" e "consumir apenas o caldo do feijão", com $64 \%$ (n=36) e 37\% (n=21) de adesão, respectivamente, pode estar contribuindo para o principal sintoma relatado pelos pacientes, a flatulência $\left(85 \%\right.$; n=38). São José ${ }^{23}$ ressalta que os alimentos ricos em enxofre podem causar desconfortogástrico, como flatulência, pelo alto teor de compostos sulfurados. 
Outra orientação que apresentou baixa adesão $(52 \%, n=29)$ foi a de evitar o excesso de café e chimarrão, pois a cafeína é um potente estimulante da contração colônica, e se consumida em quantidades excessivas pode causar diarreia. ${ }^{24}$

Quanto ao consumo de leite e derivados, somente $44 \%(n=25)$ dos pacientes diminuíram a ingestão, sendo o mesmo percentual de pacientes com intolerância à lactose. Segundo Branco, ${ }^{17} \mathrm{a}$ redução do consumo de alimentos com lactose na fase aguda é importante, independentemente da deficiência da lactase, pois sua quantidade no organismo diminui com lesão celular e com a presença de diarreia, aumentando o volume de resíduos produzidos pelo leite e, consequentemente, o desconforto do paciente.

Estudo realizado por White \& Marin-Leon ${ }^{25}$ avaliou o cumprimento de orientações nutricionais fornecidas a idosos hipertensos em serviços de saúde e verificou que, apesar de receberem orientações nutricionais, grande parte não as seguia, provavelmente por não entender as orientações recebidas e/ou se sentir desmotivado para cumpri-las e não compreender a importância das medidas para o controle das doenças. Os autores ressaltam também a necessidade de estimular o empoderamento dos pacientes mediante educação em grupos, que possa permitir autonomia nas escolhas, decisões e comportamentos frente ao tratamento.

Para Zanetti e colaboradores, ${ }^{26}$ é fundamental investigar qual as contribuições das variáveis sociodemográficas na adesão ao plano alimentar, sendo que no presente estudo destaca-se que a adesão às orientações não apresentou associação estatisticamente significativa com a escolaridade $(p=0,808)$, trabalho $(p=0,080)$ ou gênero $(p=0,813)$.

De modo geral, as orientações nutricionais realizadas aos pacientes atendidos no ambulatório de DIIs proporcionam uma dieta balanceada e almejam encorajar os hábitos alimentares adequados, tanto para pacientes na fase ativa, aliviando os sintomas, quanto para aqueles que estão em remissão da doença. Mas sabe-se que o aconselhamento e a educação nutricional são processos de intervenção longos, que demandam tempo e dedicação do profissional, pois partem da reconstrução de informações advindas dos conhecimentos e da experiência do próprio indivíduo, no processo de formação dos seus hábitos e modificação do seu comportamento. ${ }^{27}$

\section{Conclusão}

Os resultados obtidos na presente pesquisa mostraram-se relevantes quanto à percepção do paciente com DII em relação à dieta que deve ser seguida para melhora do estado nutricional, sobretudo durante a fase ativa da doença. Verificou-se adesão ao tratamento dietoterápico pela maioria dos pacientes, com melhor aceitação para as seguintes orientações "diminuir o consumo 
de gorduras e frituras", "evitar alimentos irritativos da mucosa" e "evitar o consumo de produtos industrializados", enquanto que as que apresentaram mais dificuldade de adesão foram "consumir somente o caldo do feijão", "diminuir o consumo de leite e derivados" e "evitar o consumo excessivo de café e chimarrão".

Destaca-se que os indivíduos que melhoraram o IMC e tiveram a percepção de que o acompanhamento nutricional auxiliou no tratamento da DII aderiram em média a 7,61 e 7,69 das 11 orientações, respectivamente. Observou-se, ainda, que houve redução da desnutrição, mas o excesso de peso aumentou, fazendo-se necessário um acompanhamento nutricional mais efetivo na fase de remissão da doença.

A comparação dos resultados obtidos com os encontrados na literatura foi limitada, considerando a escassez de estudos que relatam conduta dietética em doenças inflamatórias intestinais e adesão a elas; desta forma, sugere-se que mais investigações sobre o tema sejam realizadas.

Diante do exposto, é possível verificar a importância da adesão e percepção dos pacientes quanto às orientações nutricionais, oferecendo assim subsídios para que os profissionais envolvidos possam refletir sobre a conduta dietoterápica, buscar novas formas de motivação a adesão do tratamento e consequente qualidade de vida do paciente.

\section{Referências}

1. Torres JAP, Santana RM, Torres FAP, Moura AR, Torres Neto JR. Doenças inflamatórias intestinais no Hospital Universitário da Universidade Federal de Sergipe: manifestações extraintestinais. Revista Brasileira de Coloproctologia 2011; 31(2):115-119.

2. Fernandes A, Bacalhau S, Cabral J. Doença inflamatória intestinal pediátrica: uma patologia em crescimento?. Revista Acta Médica Portuguesa 2011; 24(S2):333-338.

3. Diestel CF, Santos MC, Romi MD. Tratamento nutricional nas doenças inflamatórias intestinais. Revista do Hospital Universitário Pedro Ernesto, UERJ 2012; 11(4):52-58.

4. Souza MM, Barbosa DA, Espinosa MM, Belasco AGS. Qualidade de vida de pacientes portadores de doençainflamatória intestinal. Acta Paulista de Enfermagem 2011; 24(4):479-484.

5. Souza MM, Belasco AGS, Aguilar-Nascimento JE. Perfil epidemiológico dos pacientes portadores de doença inflamatória intestinal do estado de Mato Grosso. Revista Brasileira de Coloproctologia 2008; 28(3):324-328.

6. Salviano FN, Burgos MGPA, Santos EC. Perfil socioeconômico e nutricional de pacientes com doença inflamatória intestinal internados em um hospital universitário. Arquivos de Gastroenterologia 2007; 44(2):99-106. 
7. Brasil. Ministério da Saúde. Orientações para a coleta e análise de dados antropométricos em serviços de saúde: Norma Técnica do Sistema de Vigilância Alimentar e Nutricional - SISVAN. Brasília: Ministério da Saúde; 2011.

8. Silva AF, Schieferdecker MEM, Amarante HMBS. Ingestão alimentar em pacientes com doença inflamatória intestinal. Arquivos Brasileiros de Cirurgia Digestiva 2011; 24(3):204-209.

9. Oliveira FM, Emerick APC, Soares EG. Aspectos epidemiológicos das doenças intestinais inflamatórias na macrorregião de saúde leste do Estado de Minas Gerais. Ciên. Saúde Coletiva 2010; 15(1):1031-1037.

10. Alves RF, Silva RP, Ernesto MV, Lima AG, Souza FM. Gênero e saúde: o cuidar do homem em debate. Revista Psicologia: teoria e prática 2011; 13(3):152-166.

11. Campos MOB, Cavalcante TB. Smoking: etiologic factor for Crohn's diasease. Revista de Enfermagem da UFPI 2012; 1(3): 201-204.

12. Silva AF, Schieferdecker MEM, Rocco CS, Amarante HMBS. Relação entre estado nutricional e atividade inflamatória em pacientes com doença inflamatória intestinal. Arquivos Brasileiros de Cirurgia Digestiva 2010; 23(3):154-158.

13. Boutros M, Maron D. Inflammatory bowel disease in the obese patient. Clinics in Colon and Rectal Surgery 2011; 24(4):244-252.

14. Israeli E, Graff LA, Clara I, Walker JR, Lix LM, Targownik LE, et al. Low prevalence of disability among patients with inflammatory bowel diseases a decade after diagnosis. Clinical Gastroenterology and Hepatology 2014; 12(1):1330-1337.

15. Menezes CA. Intolerância à lactose em pacientes com doença inflamatória intestinal [dissertação]. Salvador: Universidade Federal da Bahia; 2013.

16. Busnello FM, Bodanese LC, Pellanda LC, Santos ZEA. Intervenção nutricional e o impacto na adesão ao tratamento em pacientes com síndrome metabólica. Arquivos Brasileiros de Cardiologia 2011; 97(3):217-224.

17. Branco MC. Aspetos nutricionais da doença inflamatória do intestino [Monografia] Porto: Universidade do Porto; 2012.

18. Ddine LC, Ddine CC, Rodrigues CCR, Kirsten VR, Colp E. Fatores associados com a gastrite crônica em pacientes com presença ou ausência do Helicobacterpylori. Arquivos Brasileiros de Cirurgia Digestiva 2012; 25(2):96-100.

19. Santos GM, Silva LR, Santana GO. Repercussões nutricionais em crianças e adolescentes na presença de doenças inflamatórias intestinais. Revista Paulista de Pediatria 2014; 32(4):403-411.

20. Pereira LM, Vieira ALS, Horta PM, Santos LC. Fracionamento da dieta e o perfil nutricional e de saúde de mulheres. Revista de Nutrição 2014; 27(1):15-23.

21. Santos SMR. Doença de Crohn: etiopatogenia, aspetos clínicos, diagnóstico e tratamento [Dissertação]. Porto: Universidade Fernando Pessoa, Curso de Ciências Farmacêuticas; 2013.

22. Almeida LB, Marinho CB, Souza CS, Cheib VBP. Disbiose intestinal. Revista Brasileira de Nutrição Clínica 2009; 24(1):58-65. 
23. São José JFB. Avaliação qualitativa de cardápios em uma unidade de alimentação e nutrição localizada em Vitória-ES. DEMETRA: Alimentação, Nutrição \& Saúde 2014; 9(4):975-984.

24. Amarante D. Aspectos nutricionais na população de pacientes com síndrome do intestino irritável atendidos no Hospital das Clínicas da Faculdade de Medicina da Universidade de São Paulo [Dissertação]. São Paulo: Universidade de São Paulo; 2013.

25. White HJ, Marín-León L. Orientações nutricionais em serviços de saúde-a percepção de idosos portadores de hipertensão arterial e diabetes mellitus. Demetra: Alimentação, Nutrição \& Saúde 2015; 9(4):867-880.

26. Zanetti ML, Arrelias CCA, Franco RC, Santos MA, Rodrigues FFL, Faria HTG. Adesão às recomendações nutricionais e variáveis sociodemográficas em pacientes com diabetes mellitus. Rev. Esc. Enferm. USP 2015; 49(4):0619-0625.

27. Nunes, RM. Avaliação da adesão terapêutica nutricional e sua relação com os modelos de mudança do comportamento alimentar. HU Revista 2015; 40 (3 e 4):221-229.

Recebido: 16/10/2015

Revisado: $14 / 3 / 2016$

Aceito: 26/4/2016 